\title{
Gleichheit ohne Brüderlichkeit
}

\section{Die Behindertenkonvention der Vereinten Nationen und die berufliche Teilhabe}

\author{
Wolfgang Trunk
}

\begin{abstract}
Das Übereinkommen der Vereinten Nationen über die Rechte von Menschen mit Behinderungen gilt als neue Leitlinie der Behindertenpolitik auch in Deutschland. Mit der Behindertenrechtskonvention wird Behinderung nicht länger primär unter medizinischen oder sozialen Blickwinkeln betrachtet, sondern Behinderung soll als Menschenrechtsthema anerkannt werden. Die Konsequenzen für die Behindertenarbeit können jedoch auch kritisch gesehen werden.
\end{abstract}

Im Februar 2009 hat die Bundesregierung das »Übereinkommen über die Rechte von Menschen mit Behinderungen « der Vereinten Nationen ratifiziert. Seither wird diese Konvention als Leitlinie für den behindertenpolitischen Fortschritt propagiert; auch in der sozialpolitischen Debatte wird sie von allen Seiten bemüht. Bei näherem Hinsehen ergeben sich allerdings Zweifel an der fachlichen und politischen Substanz des Textes. Es steht zu befürchten, dass die Konvention in Deutschland dazu beiträgt, das Handlungsfeld der Behindertenarbeit zu desorientieren und den weiteren Sozialabbau zu bemänteln.

Die UNO-Konvention erklärt zwar die »volle und wirksame Teilhabe an der Gesellschaft « zum Zweck aller behindertenpolitischen Bemühungen (Art. 3), der Bereich "Arbeit und Beschäftigung " wird aber als ein separates und untergeordnetes Thema behandelt (Art. 27). Damit verkennt die Konvention die Bedeutung, die das Berufsleben für die Persönlichkeit hat. Fortschrittliche Politik für die Behinderten in Deutschland muss die berufliche

Wolfgang Trunk führt die Geschäfte bei der Landesarbeitsgemeinschaft der Werkstätten für behinderte Menschen in Hessen e.V. E-Mail info@lag-werkstaetten.de
Teilhabe als Hebel der sozialen Integration behandeln.

Die Probleme der Behinderten werden in der Konvention auf eine allgemeine Tendenz der »Diskriminierung « zurückgeführt (Art. 5); deshalb komme es zentral darauf an, ein »Bewusstsein « für die Belange der Behinderten zu schaffen (Art. 8). Mit dieser Position werden die gesellschaftlichen Interessen als bestimmende Faktoren ausgeblendet. Wenn behinderte Mitbürger in Deutschland benachteiligt sind, dann liegt das nicht primär an Defiziten des Bewusstseins, sondern an handfesten Interessen. Der Kern der Benachteiligung ist darin zu sehen, dass sich behinderte Personen nur eingeschränkt an der ökonomischen Wertschöpfung beteiligen können, und dass sie auch nur eingeschränkt konsumfähig sind. Dieser Umstand führt dazu, dass das Interesse an einem Einbezug der Behinderten in die Gesellschaft gering ist. Fortschrittliche Behindertenpolitik muss deshalb vor allem das Wertgesetz überwinden; die Arbeitsverhältnisse von Behinderten dürfen nicht vom Prinzip des Äquivalententauschs bestimmt sein. Behinderte müssen selbst dann über die Möglichkeit einer beruflichen Teilhabe verfügen, wenn ihr Beitrag zur Wertschöpfung unter den Kosten ihrer persönlichen Reproduktion liegt.

Im Mittelpunkt der Konvention stehen die »Menschenrechte und Grundfreiheiten « (Art. 2); die Hauptlosung der Konvention ist die "Gleichberechtigung « (Art. 5). Dieser Ansatz verfehlt die spezifischen Bedarfslagen der Behinderten. Behinderte brauchen besondere Angebote und Maßnahmen, um ihre soziale Teilhabe realisieren zu können. Mit einer bloßen Gleichberechtigung ist ihnen nicht gedient; Freiheit und Gleichheit werden erst auf der Ebene der Brüderlichkeit wirksam.

Für Behinderte wird nicht das Recht auf Arbeit, sondern nur »das gleiche Recht auf Arbeit« gefordert (Art. 27;
Hervorhebung der Autor); diese Forderung ist für die soziale Situation der Behinderten in Deutschland praktisch ohne Bedeutung. Da in Deutschland das allgemeine Recht auf Arbeit nicht normiert ist, bedeutet » Gleichberechtigung « hier, dass es auch für die Behinderten kein Recht auf Arbeit geben muss. Es wäre aber ein Fortschritt, wenn man den Behinderten das Recht auf Arbeit zubilligen würde, zumal sie noch weniger als Andere in der Lage sind, die destruktiven Wirkungen zu bewältigen, die mit einem Ausschluss von der Berufstätigkeit einhergehen.

Die Konvention ignoriert den Kausalzusammenhang von Arbeit, Krankheit und Behinderung, der in Deutschland für die Masse der Behinderungen verantwortlich ist. Nur am Rande wird bemerkt, dass es Personen gibt, »die während der Beschäftigung eine Behinderung erwerben« (Art. 27). Zwar fordert die Konvention allgemein den "Schutz der Unversehrtheit der Person" (Art. 17), aber im Zeichen der "Gleichberechtigung « wird diese Forderung nicht spezifiziert.

Auch die Ausführungen zum Thema Gesundheit beschränken sich im Wesentlichen auf die Forderung, dass die medizinische Versorgung der Behinderten »von gleicher Qualität « sein soll wie jene der Nicht-Behinderten (Art 25). Der wichtigste Beitrag zur Verbesserung der Situation wäre jedoch ein konsequenter Gesundheitsschutz in der Arbeitswelt, der verhindert, dass Beschäftigte durch anhaltende Arbeitsbelastungen Behinderungen erwerben, die schließlich dazu führen, dass sie ihr Arbeitsleben vorzeitig beenden müssen.

Die Konvention verengt den Blick auf jenes Segment der Behinderten, deren berufliche Teilhabe durch Maßnahmen der Eingliederung in reguläre Beschäftigung gefördert werden kann (Art. 27). In Deutschland brauchen die meisten Behinderten im erwerbsfähigen Alter jedoch keine Maßnahmen zur Eingliederung, 
denn entweder gehen sie noch einer regulären Tätigkeit als Arbeitnehmer nach oder sie haben ihr Erwerbsleben bereits hinter sich, weil sie vor dem Erreichen der Altersgrenze ausgegliedert worden sind. Für die Behinderten, die aktuell noch Arbeit haben, müssen Maßnahmen der Beschäftigungssicherung ergriffen werden; die bereits Ausgegliederten brauchen Angebote des Zuverdienstes. Was die nicht erwerbsfähigen Behinderten in den Werkstätten angeht, so gibt es dort nur wenige Grenzfälle, die für eine Eingliederung in reguläre Beschäftigung geeignet sind; gefragt ist hier vor allem die soziale Absicherung der Werkstätten für behinderte Menschen.

In der Konvention wird die berufliche Teilhabe der nicht erwerbsfähigen Behinderten weder als Erfordernis benannt noch als Möglichkeit erwähnt; Werkstätten für Behinderte können vor diesem Hintergrund als verzichtbar erscheinen. Diesen Ball fängt die Sozialpolitik in Deutschland auf, wenn sie Werkstätten als Sonderstruktur interpretiert, die neben der normalen Gesellschaft existiert, und die dazu beiträgt, Behinderte auszusondern, statt sie in die Gesellschaft einzubeziehen. Die wahre Integration sei nur dann gegeben, wenn sich Behinderte als Individuen in den regulären Strukturen der Gesellschaft bewegen können, und wenn sie dort nicht aus-, sondern eingeschlossen sind. Es gelte deshalb, die allgemeinen Sozialräume »inclusiv « zu gestalten und Sonderstrukturen zurückzudrängen.

Das Schlagwort von der "Inclusion " (Art. 3, engl.) kam der Sozialpolitik gerade recht, um den Anlass für ein großangelegtes Vorhaben des Sozialabbaus zu liefern. Im Herbst 2009 hat die Arbeitsund Sozialministerkonferenz von der Bundesregierung ein Gesetz zur »Re-

\section{Tipp}

Die 40-seitige deutsche Fassung des Übereinkommens über die Rechte von Menschen mit Behinderungen der Vereinten Nationen steht auf der Website des Beauftragten der Bundesregierung für die Belange der Behinderten zum kostenlosen Herunterladen zur Verfügung.

Internet http://www.alle-inklusive.behindertenbeauftragte.de form « der Eingliederungshilfe verlangt. Hintergrund sind die Fallzahlen im Bereich der Behindertenhilfe, die derzeit noch steigen, weil sich die Altersstruktur der Behinderten aufgrund der sogenannten Euthanasie-Politik des deutschen Faschismus nur langsam normalisiert.

Mit den Fallzahlen steigen die absoluten Kosten, wobei allerdings zu beachten ist, dass die Quote der sozialen Transferleistungen stabil bleibt. Im laufenden Jahr werden jedenfalls etwa 14 Milliarden Euro aufgewandt; sie machen den größten Brocken der kommunalen Sozialhilfeleistungen aus, und diese Kosten will man begrenzen und nach Möglichkeit verringern.

Hauptinstrument der Kostendämpfung soll der » personenzentrierte Ansatz « sein, mit dem die Finanzierung der Hilfen an Einzelleistungen gebunden wird, über deren Notwendigkeit wiederum von Fall zu Fall entschieden wird. Zur Kostentransparenz will man ein bundesweites Bedarfsfeststellungsverfahren einführen, mit dem die Gesamtheit der Klientel erfasst werden kann, und das die konkreten Einsparpotenziale offenbaren soll. Über ein »Teilhabemanagement « sollen die Hilfeempfänger in diese Strategie eingebunden werden.

Es bleibt zu fragen, wie man ein solches Vorhaben mit der Würde der behinderten Mitbürger vereinbaren will, von der auch die UNO-Konvention spricht (Art. 1).

\section{Aktuelle Neuerscheinung}

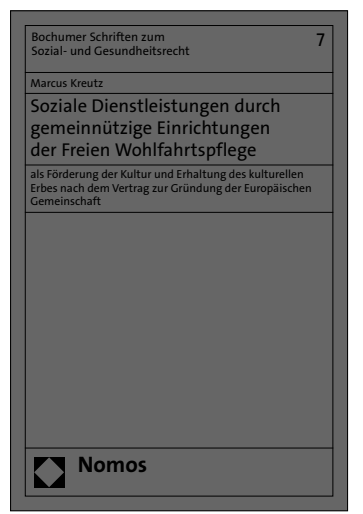

Soziale Dienstleistungen durch gemeinnützige Einrichtungen der Freien Wohlfahrtspflege

als Förderung der Kultur und Erhaltung des kulturellen Erbes nach dem Vertrag zur Gründung der Europäischen Gemeinschaft Von RA Dr. Marcus Kreutz, LL.M. 2010, 362 S., brosch., 85,-€, ISBN 978-3-8329-5418-5 (Bochumer Schriften zum Sozialund Gesundheitsrecht, Bd. 7)

Nationale Privilegien, die der Gesetzgeber gemeinnützigen Körperschaften gewährt, sind nicht zwingend als verbotene Beihilfe im Sinne von Art. 87 Abs. 1 EGV zu qualifizieren. Vielmehr ergibt sich, dass diese Privilegien als Kultur und Erhaltung des kulturellen Erbes über Art. 87 Abs. 3 lit.d) EGV rechtfertigungsfähig sind.

\section{Nomos}

Bitte bestellen Sie im Buchhandel oder versandkostenfrei unter $\downarrow$ www.nomos-shop.de 